\title{
An enhanced Craig-Bampton method
}

\author{
Jin-Gyun $\mathrm{Kim}^{1}$ and Phill-Seung Lee ${ }^{2, *, \dagger}$ \\ ${ }^{1}$ Mechanical Systems Safety Research Division, Korea Institute of Machinery and Materials, 156 Gajeongbuk-ro, \\ Yuseong-gu, Daejeon 305-343, Korea \\ ${ }^{2}$ Division of Ocean Systems Engineering, Korea Advanced Institute of Science and Technology, 291 Daehak-ro, \\ Yuseong-gu, Daejeon 305-701, Korea
}

\begin{abstract}
SUMMARY
In this paper, we propose a new component mode synthesis method by enhancing the Craig-Bampton (CB) method. To develop the enhanced $C B$ method, the transformation matrix of the $\mathrm{CB}$ method is enhanced considering the effect of residual substructural modes and the unknown eigenvalue in the enhanced transformation matrix is approximated by using O'Callahan's approach in Guyan reduction. Using the newly defined transformation matrix, original finite element models can be more accurately approximated by reduced models. For this reason, the accuracy of the reduced models is significantly improved with a low additional computational cost. We here present the formulation details of the enhanced CB method and demonstrate its performance through several numerical examples. Copyright @ 2015 John Wiley \& Sons, Ltd.
\end{abstract}

Received 21 March 2014; Revised 13 November 2014; Accepted 22 December 2014

KEY WORDS: $\quad$ structural dynamics; finite element method; model reduction; component mode synthesis; Craig-Bampton method; dynamic substructuring

\section{INTRODUCTION}

Reduced-order modeling of large finite element (FE) models is essential in many engineering fields such as ocean, mechanical, and aerospace engineering. Component mode synthesis (CMS) methods have been widely used for FE model reductions in structural dynamics. In the CMS methods, the original large structural FE model is partitioned into smaller substructural FE models connected at interface boundary. A small proportion of substructural modes (dominant substructural modes) and interface constraint conditions are used to reduce the original structural model. Because, instead of the original large structural model, we handle the reduced model constructed using the small substructural models, CMS methods can dramatically reduce the computational cost. The accurately approximated reduced models are valuable indeed in structural systems design, system identification, and experimentally verified model development.

In the 1960s, based on Hurty and Guyan's idea $[1,2]$, Craig and Bampton proposed a CMS method referred to as the Craig-Bampton (CB) method [3]. Since then, various related studies have been performed to develop robust CMS methods [4-11]. However, the CB method is still the most popular and widely used CMS method because of its simplicity and reliability. Reviews of the CMS methods can be found in [12-14].

Using the localized Lagrange multipliers at free interface boundary, Park and Park developed the flexibility-based CMS (F-CMS) method [9], in which the accuracy of reduced models is improved by effectively retaining the contribution of residual substructural modes. The conceptual idea has been employed to construct an enhanced transformation matrix used for estimating relative

\footnotetext{
*Correspondence to: Phill-Seung Lee, Division of Ocean Systems Engineering, Korea Advanced Institute of Science and Technology, 291 Daehak-ro, Yuseong-gu, Daejeon 305-701, Korea.

${ }^{\dagger}$ E-mail: phillseung@kaist.edu
} 
eigenvalue errors in the CB method [15]. However, the enhanced transformation matrix contains an unknown eigenvalue, and thus, it is not possible to use the transformation matrix in its present form for the improvement of the original $\mathrm{CB}$ method.

In order to overcome this difficulty, we borrow O'Callahan's idea, which was originally proposed to improve Guyan reduction [16]. That is, the unknown eigenvalue is approximated using O'Callahan's approach. As a result, a new enhanced transformation matrix is obtained and, using it, the enhanced $\mathrm{CB}$ method is developed. Compared with the original $\mathrm{CB}$ method, the enhanced CB method can provide significantly improved reduced-order models with a low additional computational cost.

In the following sections, we briefly review the original $\mathrm{CB}$ method, derive the enhanced $\mathrm{CB}$ method, and present the performance of the enhanced CB method through various numerical examples: rectangular plate, hyperboloid shell, stiffened plate, and solid ring problems. The numerical results of the enhanced $\mathrm{CB}$ method are compared with the original CB and F-CMS methods.

\section{CRAIG-BAMPTON METHOD}

In the CB method, global (original) structure is partitioned by $N_{s}$ substructures as in Figure 1(b). The substructures are connected with a fixed interface at the interface boundary $\Gamma$ (Figure 1(c)). The linear dynamics equations can be expressed by

$$
\begin{gathered}
\mathbf{M}_{g} \ddot{\mathbf{u}}_{g}+\mathbf{K}_{g} \mathbf{u}_{g}=\mathbf{f}_{g} \\
\mathbf{M}_{g}=\left[\begin{array}{ll}
\mathbf{M}_{s} & \mathbf{M}_{c} \\
\mathbf{M}_{c}^{T} & \mathbf{M}_{b}
\end{array}\right], \quad \mathbf{K}_{g}=\left[\begin{array}{cc}
\mathbf{K}_{s} & \mathbf{K}_{c} \\
\mathbf{K}_{c}^{T} & \mathbf{K}_{b}
\end{array}\right], \\
\mathbf{u}_{g}=\left[\begin{array}{l}
\mathbf{u}_{s} \\
\mathbf{u}_{b}
\end{array}\right], \quad \mathbf{f}_{g}=\left[\begin{array}{l}
\mathbf{f}_{s} \\
\mathbf{f}_{b}
\end{array}\right],
\end{gathered}
$$

where $\mathbf{M}_{g}$ and $\mathbf{K}_{g}$ are the global mass and stiffness matrices, and $\mathbf{u}_{g}$ and $\mathbf{f}_{g}$ are global displacement and force vectors. The subscript $g$ denotes the global structure and $(")=d^{2}() / d t^{2}$ with the time variable $t$. The subscripts $s, b$, and $c$ denote substructures, boundary interface, and coupling matrices, respectively. Note that $\mathbf{M}_{s}$ and $\mathbf{K}_{s}$ are the block diagonal mass and stiffness matrices of substructures.

The global eigenvalue problem is

$$
\mathbf{K}_{g}\left(\boldsymbol{\phi}_{g}\right)_{i}=\lambda_{i} \mathbf{M}_{g}\left(\boldsymbol{\phi}_{g}\right)_{i}, \quad i=1,2, \ldots, N_{g},
$$

where $\lambda_{i}$ and $\left(\phi_{g}\right)_{i}$ are the eigenvalue and eigenvector calculated in the global structure, respectively, and $N_{g}$ is the number of DOFs in the global structure. Note that $\lambda_{i}$ and $\left(\phi_{g}\right)_{i}$ are the square of the $i$-th natural frequency $\left(\omega_{i}^{2}\right)$ and the corresponding mode in structural dynamics, respectively.

Using the eigenvectors obtained from Equation (2), the global displacement vector $\mathbf{u}_{g}$ is represented by

$$
\mathbf{u}_{g}=\boldsymbol{\Phi}_{g} \mathbf{q}_{g},
$$

where $\boldsymbol{\Phi}_{g}$ and $\mathbf{q}_{g}$ are the global eigenvector matrix and its generalized coordinate vector, respectively.

In the CB method, the global displacement vector $\mathbf{u}_{g}$ can be defined by

$$
\mathbf{u}_{g}=\left[\begin{array}{l}
\mathbf{u}_{s} \\
\mathbf{u}_{b}
\end{array}\right]=\mathbf{T}_{0}\left[\begin{array}{l}
\mathbf{q}_{s} \\
\mathbf{u}_{b}
\end{array}\right], \quad \mathbf{T}_{0}=\left[\begin{array}{cc}
\boldsymbol{\Phi}_{s} & -\mathbf{K}_{s}^{-1} \mathbf{K}_{c} \\
\mathbf{0} & \mathbf{I}_{b}
\end{array}\right]
$$




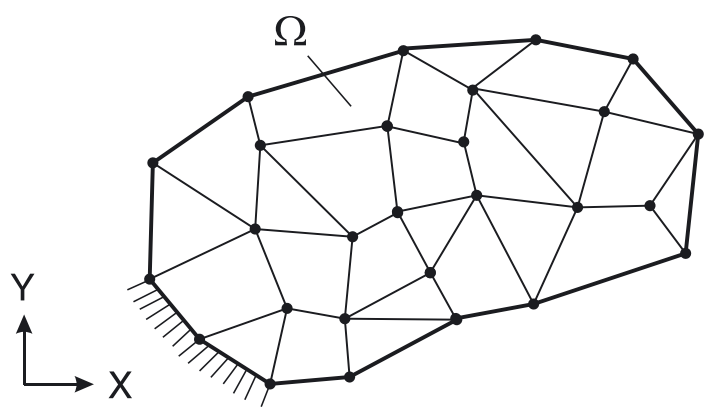

(a)

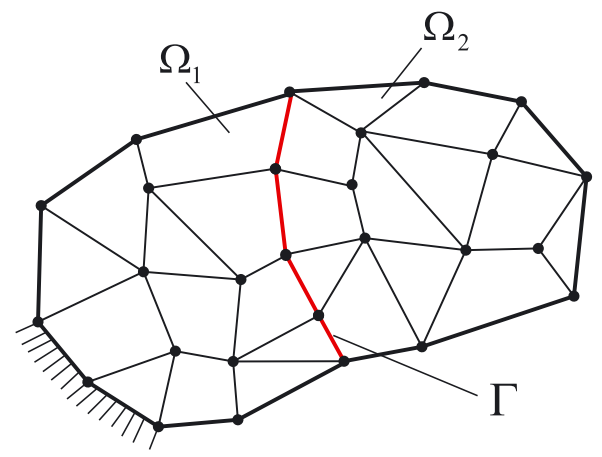

(b)
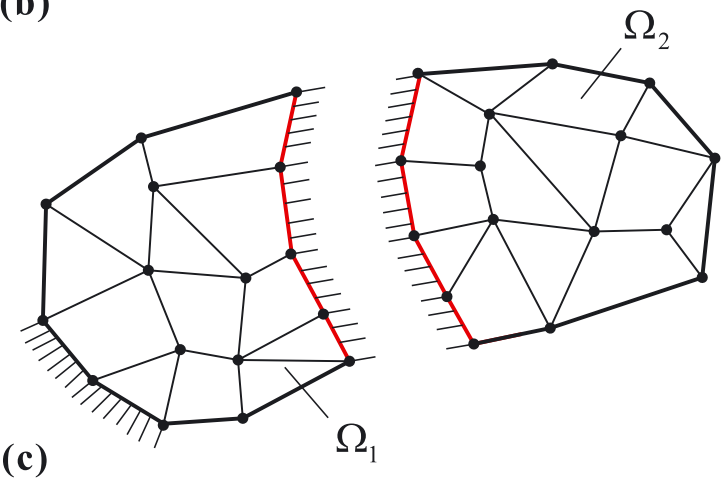

Figure 1. Global and partitioned structural models and interface handling in the Craig-Bampton method. (a) Global (non-partitioned) structure $\Omega$, (b) substructures $\Omega_{i}\left(i=1,2, \cdots, N_{S}\right)$ and interface boundary $\Gamma\left(N_{S}\right.$ denotes the number of substructure and $N_{S}=2$ in this figure), and (c) interface boundary treatment.

in which $\mathbf{q}_{s}$ is the generalized coordinate vector for the substructural modes, $\mathbf{T}_{\mathbf{0}}$ is the transformation matrix, $\mathbf{I}_{b}$ is an identity matrix for the interface boundary, and $\boldsymbol{\Phi}_{s}$ is a block diagonal eigenvector matrix calculated by the substructural eigenvalue problems

$$
\left[\mathbf{K}_{s}^{(k)}-\lambda_{i}^{(k)} \mathbf{M}_{s}^{(k)}\right]\left(\phi^{(k)}\right)_{i}=\mathbf{0}, \quad i=1,2, \ldots, N_{q}^{(k)}, \text { for } k=1,2, \ldots, N_{s},
$$

where $N_{q}^{(k)}$ is the number of deformable modes in the $k$-th substructure, and $\lambda_{i}^{(k)}$ and $\left(\phi^{(k)}\right)_{i}$ are the substructural eigenvalue and eigenvector, respectively.

The substructural displacement vector $\mathbf{u}_{s}$ can be decomposed into the dominant and residual modes

$$
\mathbf{u}_{s}=\boldsymbol{\Phi}_{s} \mathbf{q}_{s}-\mathbf{K}_{s}^{-1} \mathbf{K}_{c} \mathbf{u}_{b}=\left[\begin{array}{ll}
\boldsymbol{\Phi}_{d} & \boldsymbol{\Phi}_{r}
\end{array}\right]\left[\begin{array}{l}
\mathbf{q}_{d} \\
\mathbf{q}_{r}
\end{array}\right]-\mathbf{K}_{s}^{-1} \mathbf{K}_{c} \mathbf{u}_{b}
$$


where $\boldsymbol{\Phi}_{d}$ and $\boldsymbol{\Phi}_{r}$ are the dominant and residual substructural eigenvector matrices, respectively, and $\mathbf{q}_{d}$ and $\mathbf{q}_{r}$ are the corresponding generalized coordinate vectors. The subscripts $d$ and $r$ denote the dominant and residual terms, respectively. In general, a small number of the dominant modes is used to reduce the global structure. That is, $N_{d} \ll N_{r}$, in which $N_{d}$ and $N_{r}$ are the numbers of the dominant and residual modes, respectively.

Neglecting the residual modes in Equation (6), the global displacement vector $\mathbf{u}_{g}$ is approximated by the dominant substructural modes

$$
\mathbf{u}_{g} \approx \overline{\mathbf{u}}_{g}=\overline{\mathbf{T}}_{0}\left[\begin{array}{l}
\mathbf{q}_{d} \\
\mathbf{u}_{b}
\end{array}\right], \quad \overline{\mathbf{T}}_{0}=\left[\begin{array}{cc}
\boldsymbol{\Phi}_{d}-\mathbf{K}_{s}^{-1} \mathbf{K}_{c} \\
\mathbf{0} & \mathbf{I}_{b}
\end{array}\right]
$$

where $\overline{\mathbf{T}}_{0}$ is the reduced transformation matrix. Using $\overline{\mathbf{T}}_{0}$ in Equation (1), we can obtain the reduced equations of motion for the partitioned structure

$$
\begin{gathered}
\overline{\mathbf{M}}_{p} \ddot{\overline{\mathbf{u}}}_{p}+\overline{\mathbf{K}}_{p} \overline{\mathbf{u}}_{p}=\overline{\mathbf{f}}_{p}, \\
\overline{\mathbf{M}}_{p}=\overline{\mathbf{T}}_{0}^{T} \mathbf{M}_{g} \overline{\mathbf{T}}_{0}, \quad \overline{\mathbf{K}}_{p}=\overline{\mathbf{T}}_{0}^{T} \mathbf{K}_{g} \overline{\mathbf{T}}_{0}, \\
\overline{\mathbf{u}}_{p}=\left[\begin{array}{l}
\mathbf{q}_{d} \\
\mathbf{u}_{b}
\end{array}\right], \quad \overline{\mathbf{f}}_{p}=\overline{\mathbf{T}}_{0}^{T}\left[\begin{array}{c}
\mathbf{f}_{s} \\
\mathbf{f}_{b}
\end{array}\right],
\end{gathered}
$$

where $\overline{\mathbf{M}}_{p}$ and $\overline{\mathbf{K}}_{p}$ are the reduced mass and stiffness matrices, respectively, and $\overline{\mathbf{u}}_{p}$ and $\overline{\mathbf{f}}_{p}$ are the approximated displacement and force vectors, respectively. The subscript $p$ denotes the partitioned structure and an overbar $\left(^{-}\right)$denotes the approximated quantities.

From Equation (8), the reduced eigenvalue problem is obtained

$$
\overline{\mathbf{K}}_{p}\left(\overline{\boldsymbol{\phi}}_{p}\right)_{i}=\bar{\lambda}_{i} \overline{\mathbf{M}}_{p}\left(\overline{\boldsymbol{\phi}}_{p}\right)_{i}, \quad i=1,2, \ldots, \bar{N}_{p},
$$

in which $\bar{\lambda}_{i}$ and $\left(\overline{\boldsymbol{\phi}}_{p}\right)_{i}$ are the eigenvalue and eigenvector calculated in the reduced model, respectively, and $\bar{N}_{p}$ is the number of DOFs in the reduced model.

Using the eigenvectors obtained from Equation (9), the approximated displacement vector $\overline{\mathbf{u}}_{p}$ is represented by

$$
\overline{\mathbf{u}}_{p}=\overline{\boldsymbol{\Phi}}_{p} \overline{\mathbf{q}}_{p},
$$

where $\overline{\boldsymbol{\Phi}}_{p}$ and $\overline{\mathbf{q}}_{p}$ are the eigenvector matrix in the reduced model and the corresponding generalized coordinate vector, respectively.

\section{ENHANCED CRAIG-BAMPTON METHOD}

In the original CB method, to construct the reduced transformation matrix $\overline{\mathbf{T}}_{0}$ in Equation (7), the residual substructural modes are simply truncated without any consideration. However, when the residual substructural modes are properly considered, the transformation matrix can be constructed more accurately.

Using Equation (6) in Equation (4), $\mathbf{u}_{g}$ can be rewritten as

$$
\mathbf{u}_{g}=\left[\begin{array}{l}
\mathbf{u}_{s} \\
\mathbf{u}_{b}
\end{array}\right]=\mathbf{T}_{0}\left[\begin{array}{c}
\mathbf{q}_{d} \\
\mathbf{q}_{r} \\
\mathbf{u}_{b}
\end{array}\right], \quad \mathbf{T}_{0}=\left[\begin{array}{ccc}
\boldsymbol{\Phi}_{d} & \boldsymbol{\Phi}_{r} & -\mathbf{K}_{s}^{-1} \mathbf{K}_{c} \\
\mathbf{0} & \mathbf{0} & \mathbf{I}_{b}
\end{array}\right]
$$


Using Equation (11) in Equation (1), we obtain the equations of motion for the partitioned structure

$$
\begin{aligned}
& {\left[\frac{d^{2}}{d t^{2}} \mathbf{M}_{p}+\mathbf{K}_{p}\right] \mathbf{u}_{p}=\mathbf{f}_{p}, } \\
& \mathbf{M}_{p}=\mathbf{T}_{0}^{T} \mathbf{M}_{g} \mathbf{T}_{0}, \mathbf{K}_{p}=\mathbf{T}_{0}^{T} \mathbf{K}_{g} \mathbf{T}_{0}, \\
& \frac{d^{2}}{d t^{2}} \mathbf{M}_{p}+\mathbf{K}_{p}=\left[\begin{array}{ccc}
\hat{\mathbf{\Lambda}}_{d} & \mathbf{0} & \frac{d^{2}}{d t^{2}} \overline{\mathbf{M}}_{c} \\
\mathbf{0}^{T} & \hat{\boldsymbol{\Lambda}}_{r} & \frac{d^{2}}{d t^{2}} \mathbf{D} \\
\frac{d^{2}}{d t^{2}} \overline{\mathbf{M}}_{c}^{T} & \frac{d^{2}}{d t^{2}} \mathbf{D}^{T} & \hat{\mathbf{K}}_{b}+\frac{d^{2}}{d t^{2}} \hat{\mathbf{M}}_{b}
\end{array}\right], \\
& \mathbf{u}_{p}=\left[\begin{array}{c}
\mathbf{q}_{d} \\
\mathbf{q}_{r} \\
\mathbf{u}_{b}
\end{array}\right], \mathbf{f}_{p}=\mathbf{T}_{0}^{T}\left[\begin{array}{c}
\mathbf{f}_{s} \\
\mathbf{f}_{b}
\end{array}\right],
\end{aligned}
$$

where the component matrices are defined by

$$
\begin{gathered}
\hat{\mathbf{\Lambda}}_{d}=\boldsymbol{\Lambda}_{d}+\frac{d^{2}}{d t^{2}} \mathbf{I}_{d}, \boldsymbol{\Lambda}_{d}=\boldsymbol{\Phi}_{d}^{T} \mathbf{K}_{s} \boldsymbol{\Phi}_{d}, \mathbf{I}_{d}=\boldsymbol{\Phi}_{d}^{T} \mathbf{M}_{s} \boldsymbol{\Phi}_{d}, \\
\overline{\mathbf{M}}_{c}=\boldsymbol{\Phi}_{d}^{T}\left[\mathbf{M}_{c}-\mathbf{M}_{s} \mathbf{K}_{s}^{-1} \mathbf{K}_{c}\right], \\
\hat{\mathbf{\Lambda}}_{r}=\mathbf{\Lambda}_{r}+\frac{d^{2}}{d t^{2}} \mathbf{I}_{r}, \boldsymbol{\Lambda}_{r}=\boldsymbol{\Phi}_{r}^{T} \mathbf{K}_{s} \boldsymbol{\Phi}_{r}, \mathbf{I}_{r}=\boldsymbol{\Phi}_{r}^{T} \mathbf{M}_{s} \boldsymbol{\Phi}_{r}, \\
\mathbf{D}=\boldsymbol{\Phi}_{r}^{T}\left[-\mathbf{M}_{s} \mathbf{K}_{s}^{-1} \mathbf{K}_{c}+\mathbf{M}_{c}\right], \\
\hat{\mathbf{M}}_{b}=\mathbf{M}_{b}+\mathbf{K}_{c}^{T} \mathbf{K}_{s}^{-1} \mathbf{M}_{s} \mathbf{K}_{s}^{-1} \mathbf{K}_{c}-\mathbf{M}_{c}^{T} \mathbf{K}_{s}^{-1} \mathbf{K}_{c}-\mathbf{K}_{c}^{T} \mathbf{K}_{s}^{-1} \mathbf{M}_{c}, \\
\hat{\mathbf{K}}_{b}=\mathbf{K}_{b}-\mathbf{K}_{c}^{T} \mathbf{K}_{s}^{-1} \mathbf{K}_{c} .
\end{gathered}
$$

Note that Equation (12) is the original equations of motion that contain all the substructural modes.

Substituting Equation (12c) into Equation (12a) with $\mathbf{f}_{p}=\mathbf{0}$ and considering the second row in the resulting matrix equation, we obtain

$$
\mathbf{q}_{r}=-\hat{\boldsymbol{\Lambda}}_{r}^{-1}\left[\frac{d^{2}}{d t^{2}} \mathbf{D} \mathbf{u}_{b}\right] .
$$

Substituting Equation (14) into Equation (11), $\mathbf{u}_{s}$ can be represented by

$$
\mathbf{u}_{s}=\boldsymbol{\Phi}_{d} \mathbf{q}_{d}-\mathbf{K}_{s}^{-1} \mathbf{K}_{c} \mathbf{u}_{b}-\frac{d^{2}}{d t^{2}} \hat{\mathbf{F}}_{r}\left[-\mathbf{M}_{s} \mathbf{K}_{s}^{-1} \mathbf{K}_{c}+\mathbf{M}_{c}\right] \mathbf{u}_{b},
$$

Table I. Comparison between the CB and enhanced CB methods.

\begin{tabular}{lcc}
\hline & CB & Enhanced CB \\
\hline Transformation matrix & $\overline{\mathbf{T}}_{0}$ & $\overline{\mathbf{T}}_{0}+\overline{\mathbf{T}}_{r}$ \\
& & $\overline{\mathbf{M}}_{p}+\overline{\mathbf{T}}_{r}^{T} \mathbf{M}_{g} \overline{\mathbf{T}}_{0}$ \\
Reduced mass matrix & & $+\overline{\mathbf{T}}_{0}^{T} \mathbf{M}_{g} \overline{\mathbf{T}}_{r}+\overline{\mathbf{T}}_{r}^{T} \mathbf{M}_{g} \overline{\mathbf{T}}_{r}$ \\
& & $\overline{\mathbf{K}}_{p}+\overline{\mathbf{T}}_{r}^{T} \mathbf{K}_{g} \overline{\mathbf{T}}_{0}$ \\
& $+\overline{\mathbf{T}}_{0}^{T} \mathbf{K}_{g} \overline{\mathbf{T}}_{r}+\overline{\mathbf{T}}_{r}^{T} \mathbf{K}_{g} \overline{\mathbf{T}}_{r}$ \\
Reduced stiffness matrix & $\overline{\mathbf{K}}_{p}$ & $\bar{N}_{p}$ \\
Size of the reduced matrices & $\bar{N}_{p}$ & \\
\hline
\end{tabular}


with

$$
\hat{\mathbf{F}}_{r}=\boldsymbol{\Phi}_{r} \hat{\boldsymbol{\Lambda}}_{r}^{-1} \boldsymbol{\Phi}_{r}^{T}=\boldsymbol{\Phi}_{r}\left[\boldsymbol{\Lambda}_{r}+\frac{d^{2}}{d t^{2}} \mathbf{I}_{r}\right]^{-1} \boldsymbol{\Phi}_{r}^{T},
$$

where $\hat{\mathbf{F}}_{r}$ represents the residual flexibility of the substructures.

We here invoke harmonic response $\left(d^{2} / d t^{2}=-\lambda\right)$, and then $\hat{\mathbf{F}}_{r}$ can be approximated as

$$
\begin{aligned}
\hat{\mathbf{F}}_{r} & =\boldsymbol{\Phi}_{r}\left[\Lambda_{r}-\lambda \mathbf{I}_{r}\right]^{-1} \boldsymbol{\Phi}_{r}^{T} \\
& \approx \boldsymbol{\Phi}_{r} \Lambda_{r}^{-1} \boldsymbol{\Phi}_{r}^{T}+\lambda \boldsymbol{\Phi}_{r} \Lambda_{r}^{-2} \boldsymbol{\Phi}_{r}^{T}=\mathbf{F}_{r s}+\lambda \mathbf{F}_{r m},
\end{aligned}
$$

where $\mathbf{F}_{r s}$ and $\mathbf{F}_{r m}$ are the static and dynamic parts of the residual flexibility, respectively.

Using Equation (17) in Equation (15) and truncating terms higher than the order of $\lambda$, $\mathbf{u}_{s}$ can be approximated as

$$
\mathbf{u}_{s} \approx \overline{\mathbf{u}}_{s}=\boldsymbol{\Phi}_{d} \mathbf{q}_{d}-\mathbf{K}_{s}^{-1} \mathbf{K}_{c} \mathbf{u}_{b}+\lambda \mathbf{F}_{r s}\left[-\mathbf{M}_{s} \mathbf{K}_{s}^{-1} \mathbf{K}_{c}+\mathbf{M}_{c}\right] \mathbf{u}_{b},
$$

in which $\mathbf{F}_{r s}$ is indirectly calculated by subtracting the dominant flexibility matrix from the full flexibility matrix as

$$
\mathbf{F}_{r s}=\mathbf{K}_{s}^{-1}-\boldsymbol{\Phi}_{d} \Lambda_{d}^{-1} \boldsymbol{\Phi}_{d}^{T} .
$$

Using $\overline{\mathbf{u}}_{s}$ defined in Equation (18) instead of $\mathbf{u}_{s}$ in Equation (4), we finally obtain

$$
\mathbf{u}_{g} \approx \overline{\mathbf{u}}_{g}=\overline{\mathbf{T}}_{1} \overline{\mathbf{u}}_{p}, \quad \overline{\mathbf{T}}_{1}=\overline{\mathbf{T}}_{0}+\overline{\mathbf{T}}_{r},
$$

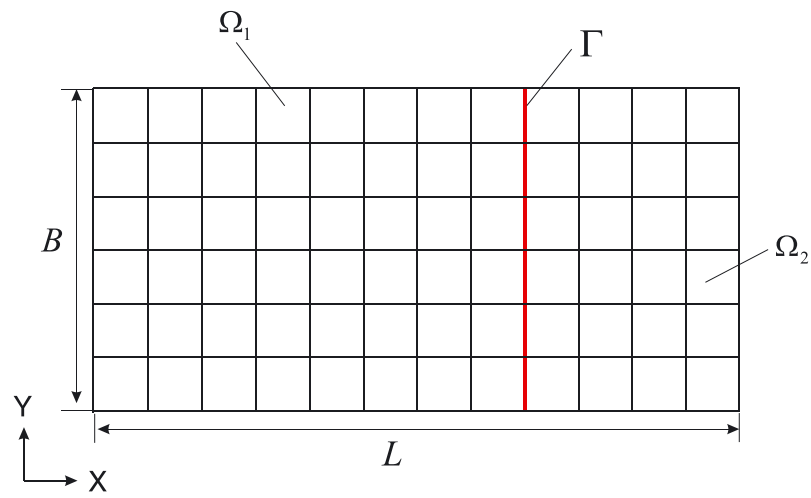

Figure 2. Rectangular plate problem.

Table II. Retained substructural mode numbers $N_{d}^{(k)}$ in the rectangular plate problem.

\begin{tabular}{lcccc}
\hline CMS & Case & $N_{d}^{(1)}$ & $N_{d}^{(2)}$ & $N_{d}$ \\
\hline CB and enhanced CB & 1 & 7 & 3 & 10 \\
& 2 & 13 & 7 & 20 \\
& 3 & 26 & 14 & 40 \\
& 4 & 52 & 28 & 80 \\
F-CMS & & 7 & 3 & 10 \\
& 1 & 13 & 7 & 20 \\
& 2 & 15 & 40 \\
& 3 & 25 & 15 & \\
& 4 & 50 & 30 & 80 \\
\hline
\end{tabular}


with

$$
\overline{\mathbf{T}}_{r}=\left[\begin{array}{ll}
\mathbf{0} & \lambda \mathbf{F}_{r s}\left[-\mathbf{M}_{s} \mathbf{K}_{s}^{-1} \mathbf{K}_{c}+\mathbf{M}_{c}\right] \\
\mathbf{0} & \mathbf{0}
\end{array}\right],
$$

where $\overline{\mathbf{T}}_{1}$ is the transformation matrix enhanced by $\overline{\mathbf{T}}_{r}$. Note that, because $\overline{\mathbf{T}}_{r}$ contains the eigenvalue $\lambda$, it is regarded as a transformation matrix related to the dynamic effect. In the previous study [15], the enhanced transformation matrix $\overline{\mathbf{T}}_{1}$ in Equation (20) is employed to develop an accurate error estimator for the CB method.

Here, a difficulty arises. Because the eigenvalue $\lambda$ in $\overline{\mathbf{T}}_{r}$ is unknown, the enhanced transformation matrix $\overline{\mathbf{T}}_{1}$ cannot be used to improve the original CB method in its present form. To handle the unknown eigenvalue $\lambda$ in $\overline{\mathbf{T}}_{r}$, we employ O'Callahan's approach, which was proposed to improve

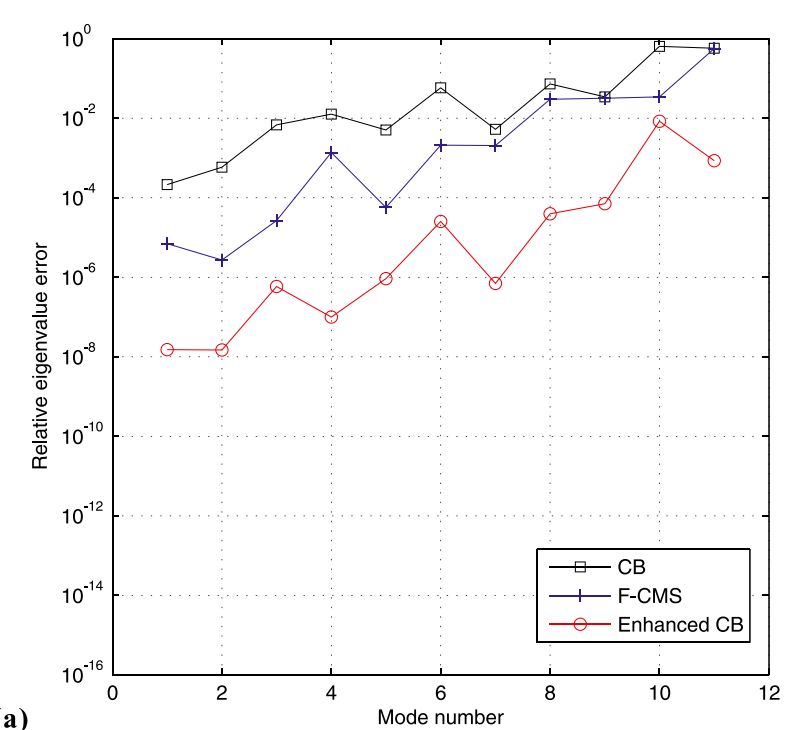

(a)

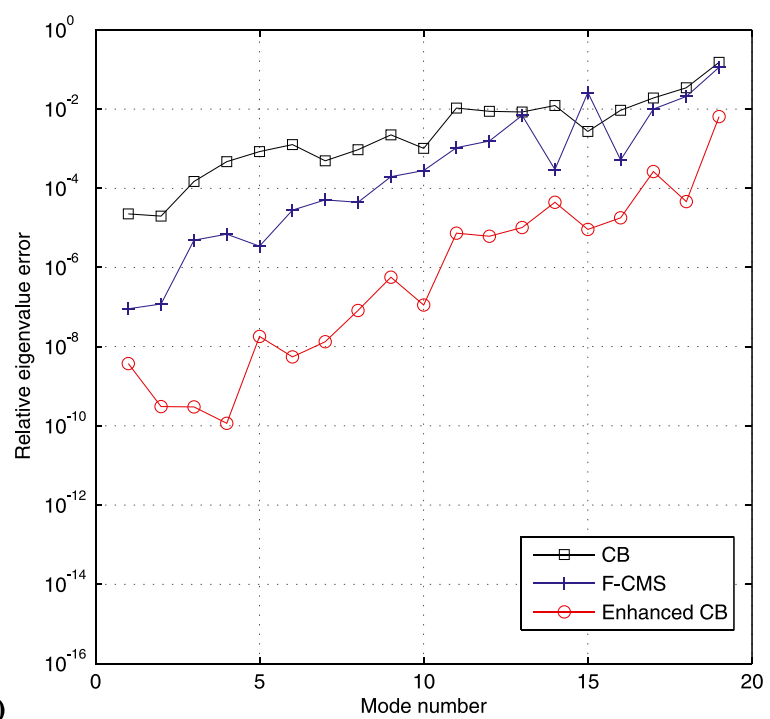

Figure 3. Relative eigenvalue errors in the rectangular plate problem. (a) $N_{d}=10$ and (b) $N_{d}=20$. 
Guyan reduction [16]. The excellent performance of O'Callahan's approach in Guyan reduction has been well known [17, 18]. From Equation (8) with $\overline{\mathbf{f}}_{p}=\mathbf{0}$ and $\ddot{\overline{\mathbf{u}}}_{p}=-\lambda \overline{\mathbf{u}}_{p}$, the following relation is obtained:

$$
\lambda \overline{\mathbf{u}}_{p}=\overline{\mathbf{M}}_{p}^{-1} \overline{\mathbf{K}}_{p} \overline{\mathbf{u}}_{p} .
$$

Using Equation (22) in Equation (20), $\overline{\mathbf{T}}_{r}$ is newly defined by

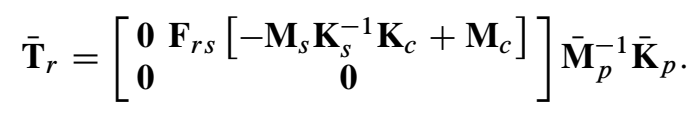

Using the redefined $\overline{\mathbf{T}}_{r}$ in Equation (20), $\overline{\mathbf{T}}_{1}$ can be expressed without the unknown eigenvalue $\lambda$. Using the enhanced transformation matrix $\overline{\mathbf{T}}_{1}$ redefined by $\overline{\mathbf{T}}_{r}$ in Equation (20), the new reduced mass and stiffness matrices denoted by tilde $(\sim)$ are defined

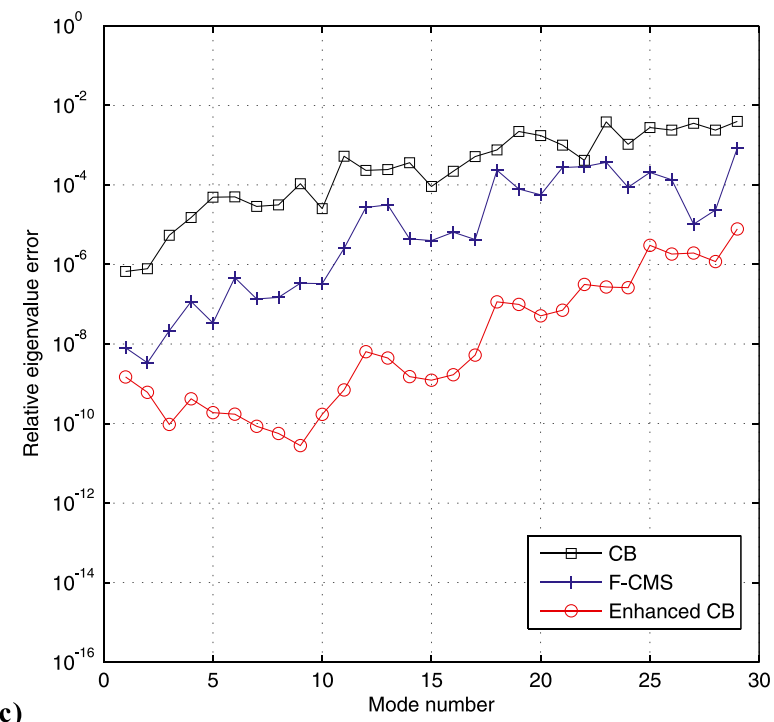

(c)

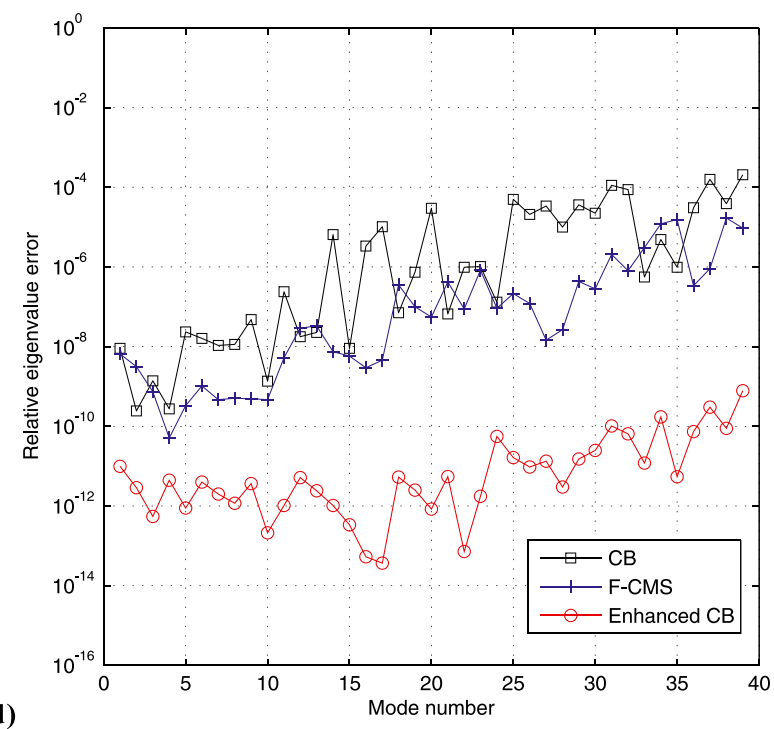

Figure 4. Relative eigenvalue errors in the rectangular plate problem. (c) $N_{d}=40$ and (d) $N_{d}=80$. 


$$
\begin{aligned}
\tilde{\mathbf{M}}_{p} & =\overline{\mathbf{T}}_{1}^{T} \mathbf{M}_{g} \overline{\mathbf{T}}_{1}=\overline{\mathbf{M}}_{p}+\overline{\mathbf{T}}_{r}^{T} \mathbf{M}_{g} \overline{\mathbf{T}}_{0}+\overline{\mathbf{T}}_{0}^{T} \mathbf{M}_{g} \overline{\mathbf{T}}_{r}+\overline{\mathbf{T}}_{r}^{T} \mathbf{M}_{g} \overline{\mathbf{T}}_{r}, \\
\tilde{\mathbf{K}}_{p} & =\overline{\mathbf{T}}_{1}^{T} \mathbf{K}_{g} \overline{\mathbf{T}}_{1}=\overline{\mathbf{K}}_{p}+\overline{\mathbf{T}}_{r}^{T} \mathbf{K}_{g} \overline{\mathbf{T}}_{0}+\overline{\mathbf{T}}_{0}^{T} \mathbf{K}_{g} \overline{\mathbf{T}}_{r}+\overline{\mathbf{T}}_{r}^{T} \mathbf{K}_{g} \overline{\mathbf{T}}_{r} .
\end{aligned}
$$

Because of the compensation of the residual substructural modes in $\overline{\mathbf{T}}_{1}$, the reduced mass and stiffness matrices in Equation (24) are more precisely constructed than the original reduced matrices in Equation (8). For this reason, when the newly defined $\tilde{\mathbf{M}}_{p}$ and $\tilde{\mathbf{K}}_{p}$ are used in Equation (9), the solution accuracy of the reduced eigenvalue problem can be improved.

Table I shows the comparison of the original and enhanced CB methods. It is important to note that both methods (original and enhanced) produce reduced models of the same size. Compared with the original CB method, the residual flexibility $\mathbf{F}_{r s}$ and the inverse of the reduced mass matrix $\overline{\mathbf{M}}_{p}^{-1}$ are additionally computed to construct the enhanced transformation matrix $\overline{\mathbf{T}}_{1}$ in the enhanced CB method.

However, $\mathbf{F}_{r s}$ can be simply calculated by reusing $\mathbf{K}_{s}^{-1}$ in Equation (4) and the dominant substructural eigensolutions in Equation (7) (Equation (19)). Also, the size of the reduced mass matrix $\overline{\mathbf{M}}_{p}$ is small because it consists of a small number of dominant substructural modes and interface DOFs. For these reasons, we can easily identify the fact that the additional computational cost of the enhanced CB method is not high.

Note that the concept of the residual flexibility $\hat{\mathbf{F}}_{r}$ in Equation (16) is essential for CMS methods with free interface boundary [9-11, 19], but the concept has not been employed for the improvement of CMS methods with fixed interface boundary (like the CB and Automated Multi-Level Substructuring (AMLS) methods) [3, 8].
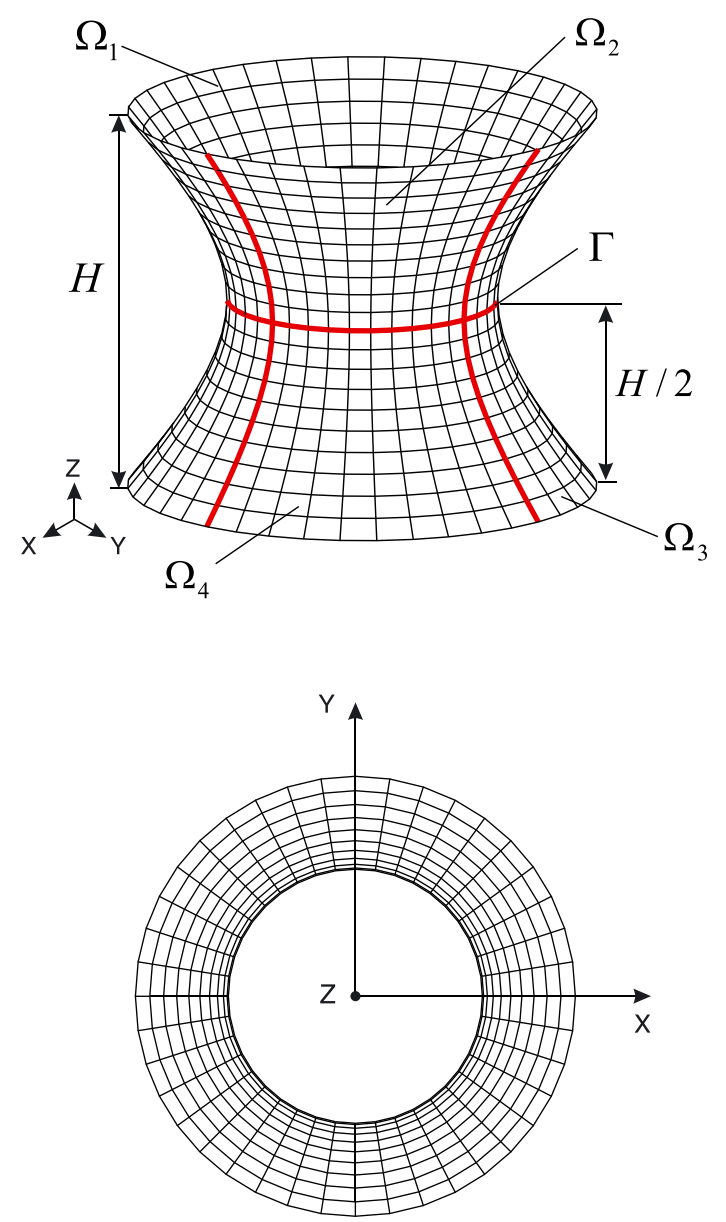

Figure 5. Hyperboloid shell problem. 


\section{NUMERICAL EXAMPLES}

In this section, we test the performance of the enhanced CB method compared with the original CB and F-CMS methods. It should be noted that, because of the use of localized Lagrange multipliers $[9,19]$, the F-CMS method generally requires more DOFs in reduced models (larger size of reduced matrices) than the original and enhanced $\mathrm{CB}$ methods for the same number of retained dominant substructural modes.

Four different structural problems are considered: rectangular plate, hyperboloid shell, stiffened plate, and solid ring problems. These are modeled by four-node Mixed Interpolation of Tensorial Components (MITC) shell (e.g., [20-23]) and eight-node brick elements. We here use the frequency cutoff mode selection method [24] to select the dominant substructural modes.

Table III. Retained substructural mode numbers $N_{d}^{(k)}$ in the hyperboloid shell problem.

\begin{tabular}{lcccccc}
\hline CMS & Case & $N_{d}^{(1)}$ & $N_{d}^{(2)}$ & $N_{d}^{(3)}$ & $N_{d}^{(4)}$ & $N_{d}$ \\
\hline CB and enhanced CB & 1 & 17 & 3 & 17 & 3 & 40 \\
& 2 & 33 & 7 & 33 & 7 & 80 \\
F-CMS & 1 & 15 & 5 & 15 & 5 & 40 \\
& 2 & 29 & 11 & 29 & 11 & 80 \\
\hline
\end{tabular}

(a)
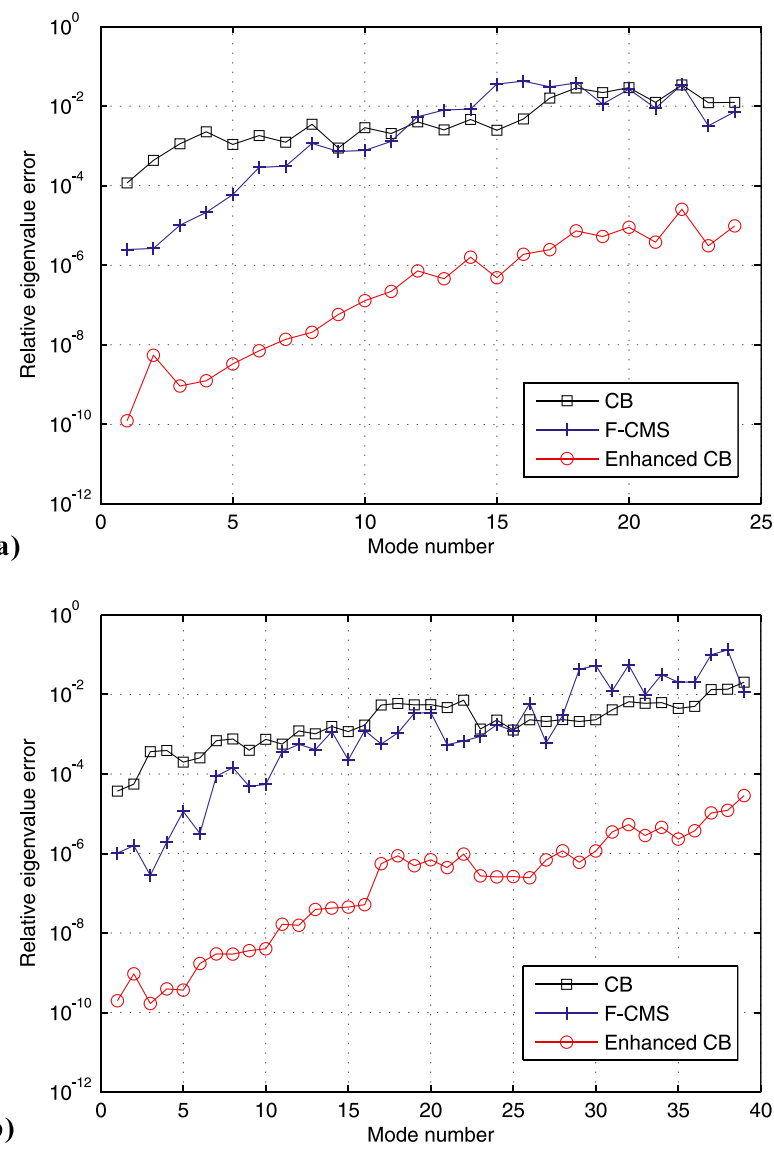

Figure 6. Relative eigenvalue errors in the hyperboloid shell problem. (a) $N_{d}=40$ and (b) $N_{d}=80$. 
The following relative eigenvalue error is used to evaluate the performance of the enhanced CB method:

$$
\xi_{i}=\frac{\bar{\lambda}_{i}-\lambda_{i}}{\lambda_{i}}
$$

in which $\xi_{i}$ denotes the relative eigenvalue error for the $i$-th mode, and $\lambda_{i}$ and $\bar{\lambda}_{i}$ are the exact and approximated eigenvalues, respectively. These eigenvalues are calculated from the global (original) and reduced eigenvalue problems (Equations (2) and (9)).

\subsection{Rectangular plate problem}

Let us consider a rectangular plate with free boundary as shown in Figure 2. Length $L$ is $0.6096 \mathrm{~m}$, width $B$ is $0.3048 \mathrm{~m}$, and thickness $h$ is $3.175 \times 10^{-3} \mathrm{~m}$. Young's modulus $E$ is $72 \mathrm{G} \mathrm{Pa}$, Poisson's ratio $v$ is 0.33 , and density $\rho_{s}$ is $2796 \mathrm{~kg} / \mathrm{m}^{3}$. The plate is modeled by a $12 \times 6$ mesh of the four-node MITC shell FEs, and the structural model is partitioned into two substructures $\left(N_{s}=2\right)$.

We consider four numerical cases with 10,20,40, and 80 dominant substructural modes selected. The numbers of retained substructural modes $N_{d}^{(k)}$ are listed in Table II. Figures 3 and 4 present the relative eigenvalue errors obtained by the original CB, F-CMS, and enhanced CB methods. The results show that the enhanced $\mathrm{CB}$ method outperforms the other two methods regardless of the number of retained substructural modes.
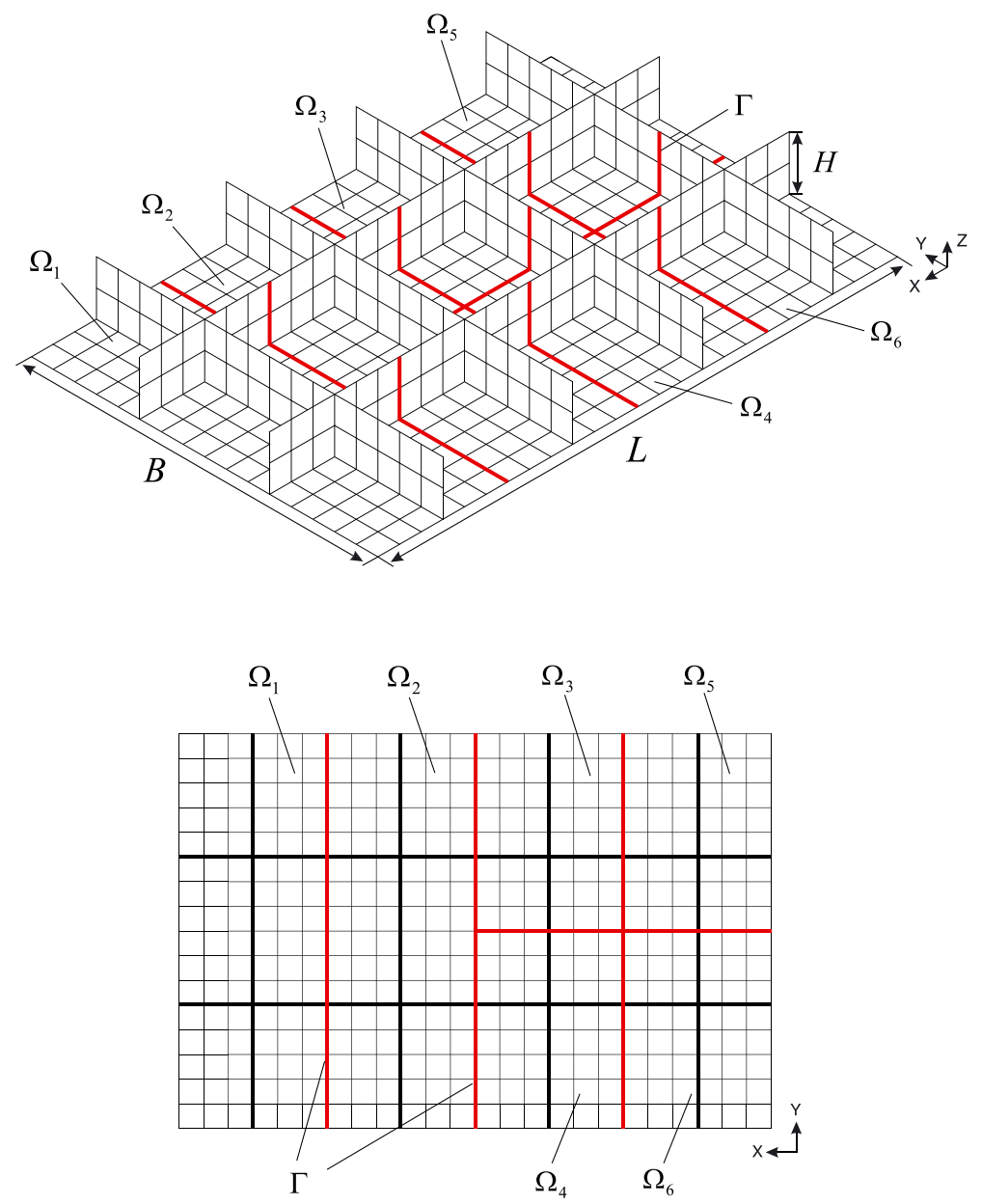

Figure 7. Stiffened plate problem. 
Table IV. Retained substructural mode numbers $N_{d}^{(k)}$ in the stiffened plate problem.

\begin{tabular}{lcccccccc}
\hline CMS & Case & $N_{d}^{(1)}$ & $N_{d}^{(2)}$ & $N_{d}^{(3)}$ & $N_{d}^{(4)}$ & $N_{d}^{(5)}$ & $N_{d}^{(6)}$ & $N_{d}$ \\
\hline \multirow{2}{*}{ CB and enhanced CB } & 1 & 20 & 8 & 3 & 3 & 8 & 8 & 50 \\
& 2 & 29 & 17 & 6 & 6 & 11 & 11 & 80 \\
F-CMS & 1 & 11 & 11 & 7 & 7 & 7 & 7 & 50 \\
& 2 & 18 & 18 & 11 & 11 & 11 & 11 & 80 \\
\hline
\end{tabular}

(a)
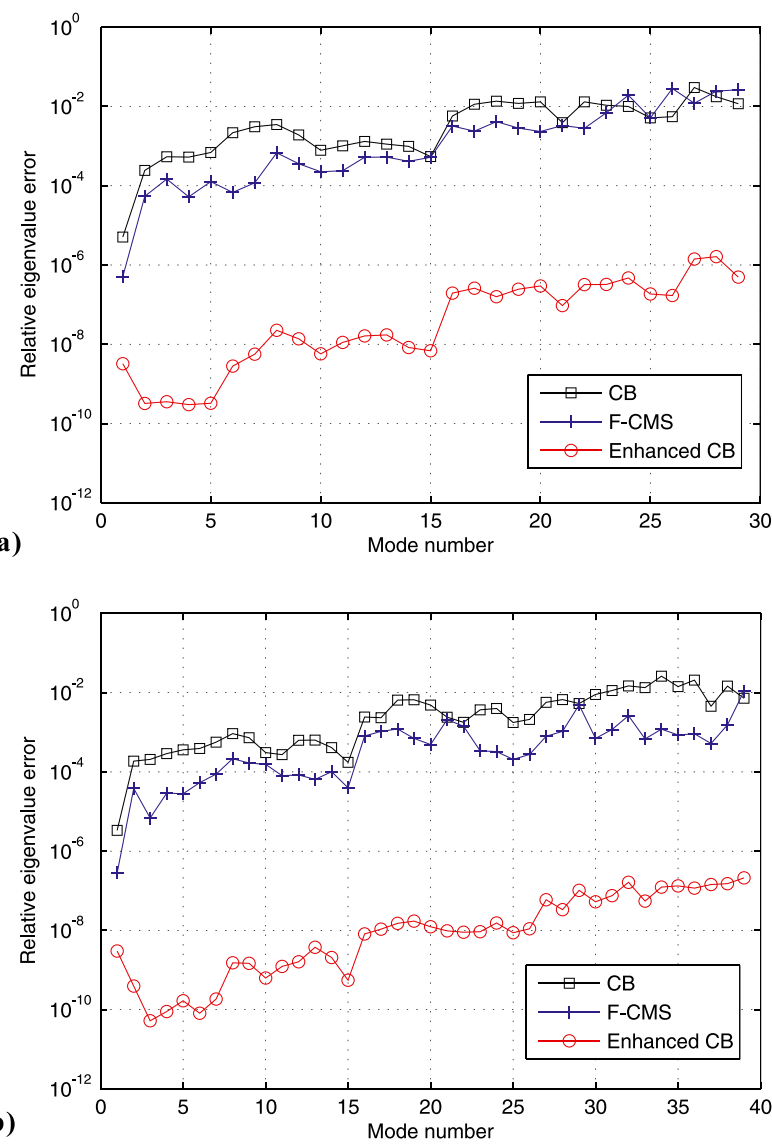

Figure 8. Relative eigenvalue errors in the stiffened plate problem. (a) $N_{d}=50$ and (b) $N_{d}=80$.

\subsection{Hyperboloid shell problem}

We here consider a hyperboloid shell structure of height $H=4.0 \mathrm{~m}$ and thickness $h=0.05 \mathrm{~m}$. Young's modulus $E$ is $69 \mathrm{GPa}$, Poisson's ratio $v$ is 0.35 , and density $\rho_{s}$ is $2700 \mathrm{~kg} / \mathrm{m}^{3}$. The midsurface of this shell structure is described by

$$
x^{2}+y^{2}=2+z^{2} ; z \in[-2,2] .
$$

We use a mesh of 20 (axial) $\times 40$ (circumferential) MITC4 shell elements (Figure 5). The FE model of the hyperboloid shell is partitioned into four substructures $\left(N_{s}=4\right)$. 

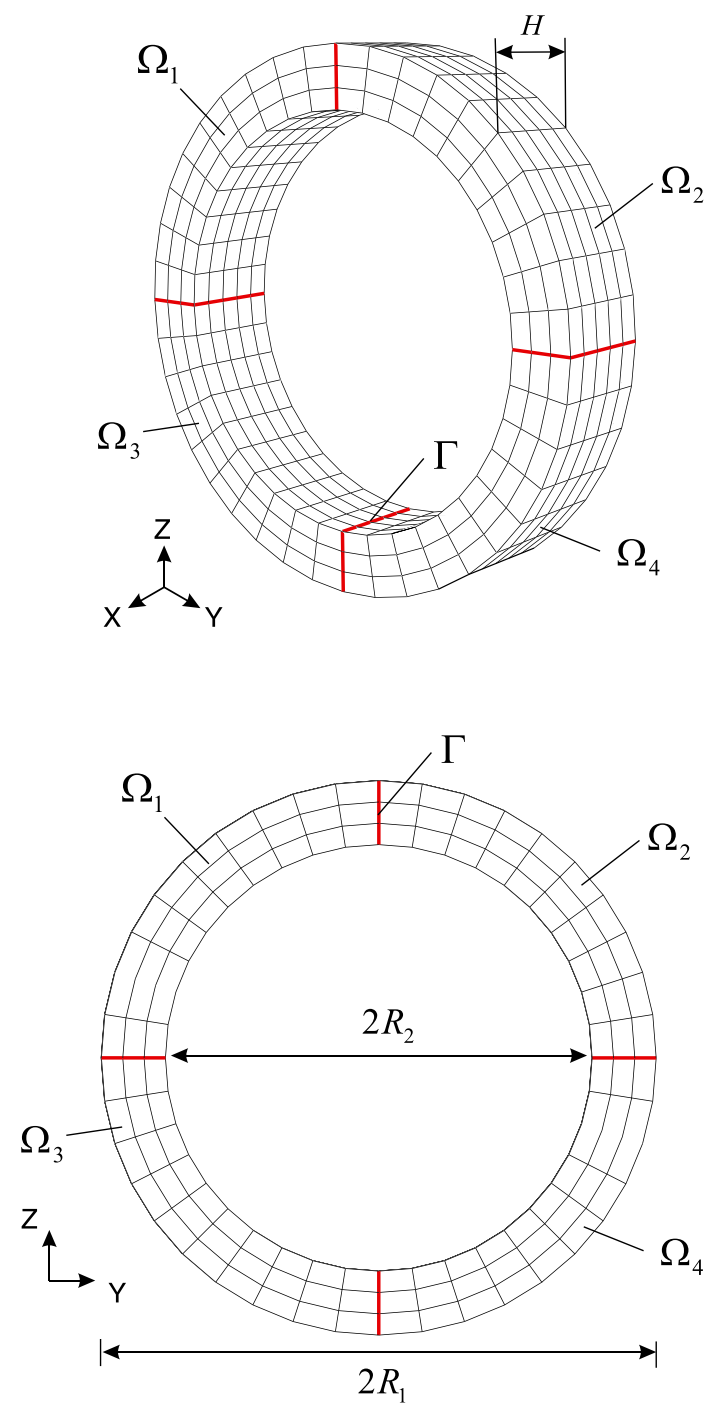

Figure 9. Solid ring problem.

We use 40 and 80 dominant substructural modes selected for two numerical cases $\left(N_{d}=40\right.$ and $N_{d}=80$ ). The numbers of dominant substructural modes $N_{d}^{(k)}$ are listed in Table III. Figure 6 shows that the enhanced CB method gives much better solution accuracy than the other two methods.

\subsection{Stiffened plate problem}

We here consider a stiffened plate with free boundary (Figure 7). Length $L$ is $4.8 \mathrm{~m}$, width $B$ is $3.2 \mathrm{~m}$, and thickness $h$ is $0.03 \mathrm{~m}$. The flat plate has two longitudinal and four transverse stiffeners, and height $H$ is $0.5 \mathrm{~m}$. Young's modulus $E$ is $210 \mathrm{GPa}$, Poisson's ratio $v$ is 0.3 , and density $\rho_{s}$ is $7850 \mathrm{~kg} / \mathrm{m}^{3}$. The bottom plate is modeled by a mesh of $24 \times 16$ shell FEs, and the longitudinal and transverse stiffeners are modeled by meshes of $24 \times 2$ and $16 \times 2$ shell FEs, respectively. This stiffened plate is partitioned into six substructures $\left(N_{s}=6\right)$.

We use 50 and 80 dominant substructural modes selected in two numerical cases $\left(N_{d}=50\right.$ and $N_{d}=80$ ), and the numbers of dominant substructural modes $N_{d}^{(k)}$ are listed in Table IV. The relative eigenvalue errors are plotted in Figure 8. The results show the robustness of the enhanced CB method. 


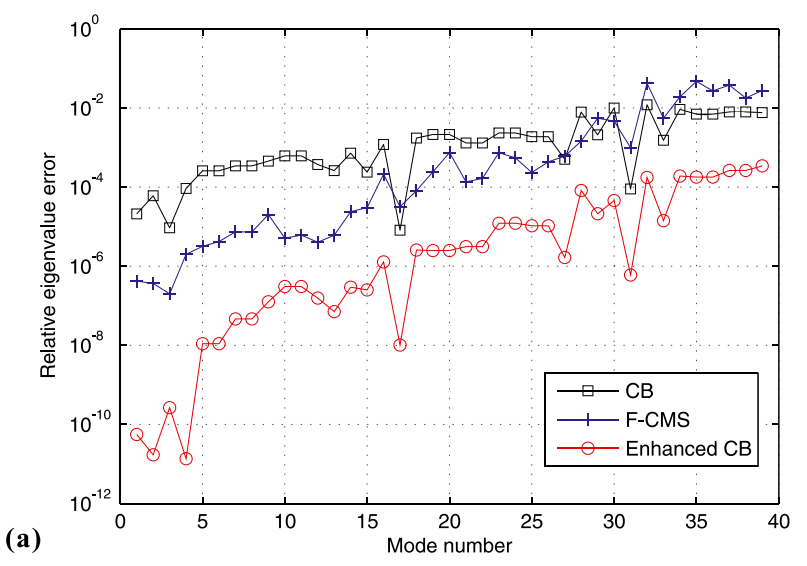

(a)

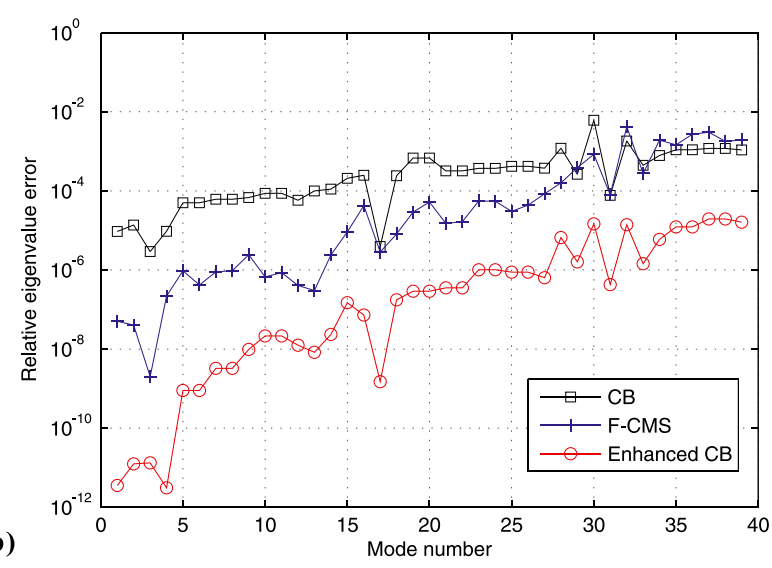

Figure 10. Relative eigenvalue errors in the solid ring problem. (a) $N_{d}=80$ and (b) $N_{d}=160$.

\subsection{Solid ring problem}

Let us consider a solid ring problem (Figure 9). Height $H$ is $0.05 \mathrm{~m}$, and the radii $R_{1}$ and $R_{2}$ are $0.13 \mathrm{~m}$ and $0.1 \mathrm{~m}$, respectively. Young's modulus $E$ is $72 \mathrm{GPa}$, Poisson's ratio $v$ is 0.33 , and density $\rho_{s}$ is $2796 \mathrm{~kg} / \mathrm{m}^{3}$. The solid ring structure is modeled by a mesh of 40 (circumferential) $\times 3$ (radial) $\times 5$ (axial) brick elements and is partitioned into four identical substructures $\left(N_{s}=4\right)$.

From each substructure, 20 and 40 dominant substructural modes are selected for two numerical cases $\left(N_{d}=80\right.$ and $\left.N_{d}=160\right)$. Figure 10 consistently demonstrates the excellent performance of the enhanced CB method.

We finally note that, when we obtain Equation (18), it is possible to contain terms of higher than order $\lambda$, and thus, the enhanced transformation matrix can be more accurate. However, we could not obtain meaningful improvement in the solution accuracy with the higher-order enhanced transformation matrix.

\section{CONCLUSIONS}

In this paper, we developed a new CMS method by improving the well-known CB method. Unlike the original $\mathrm{CB}$ method, the residual substructural modes are considered to construct the transformation matrix. As a result, the original $\mathrm{CB}$ transformation matrix is enhanced by the additional dynamic term, in which the unknown eigenvalue is approximated using O'Callahan's approach.

Using the enhanced transformation matrix, global (original) structural models can be more precisely reduced and then the accuracy of reduced models is dramatically improved with a low additional computational cost. The excellent performance of the enhanced CB method was demonstrated through various numerical examples. 
To practically use the enhanced CB method, mode selection and error estimation techniques are essential $[15,19,25]$, and also, the conceptual idea of the enhanced CB method would be used to improve other CMS methods.

\section{ACKNOWLEDGEMENTS}

This work was supported by the Basic Science Research Program through the National Research Foundation of Korea (NRF) funded by the Ministry of Education, Science and Technology (No.2014R1A1A1A05007219), and the grant NEMA-NH-2012-69 from the Natural Hazard Mitigation Research Group, National Emergency Management Agency of Korea.

\section{REFERENCES}

1. Hurty W. Dynamic analysis of structural systems using component modes. AIAA Journal 1965; 3(4):678-685.

2. Guyan R. Reduction of stiffness and mass matrices. AIAA Journal 1965; 3(2):380.

3. Craig RR, Bampton MCC. Coupling of substructures for dynamic analysis. AIAA Journal 1968; 6(7):1313-1319.

4. MacNeal RH. Hybrid method of component mode synthesis. Computers \& Structures 1971; 1(4):581-601.

5. Benfield WA, Hruda RF. Vibration analysis of structures by component mode substitution. AIAA Journal 1971; 9:1255-1261.

6. Rubin S. Improved component-mode representation for structural dynamic analysis. AIAA Journal 1975; 13(8): 995-1006.

7. Hintz RM. Analytical methods in component modal synthesis. AIAA Journal 1975; 13(8):1007-1016.

8. Bennighof JK, Lehoucq RB. An automated multilevel substructuring method for eigenspace computation in linear elastodynamics. SIAM Journal on Scientific Computing 2004; 25(6):2084-2106.

9. Park KC, Park YH. Partitioned component mode synthesis via a flexibility approach. AIAA Journal 2004; 42(6):1236-1245.

10. Rixen DJ. A dual Craig-Bampton method for dynamic substructuring. Journal of Computational and Applied Mathematics 2004; 168(1-2):383-391.

11. Markovic D, Park KC, Ibrahimbegovic A. Reduction of substructural interface degrees of freedom in flexibility-based component mode synthesis. International Journal of Numerical Methods in Engineering 2007; 70:163-180.

12. Craig RR. Coupling of substructures for dynamic analyses: an overview. in: Proceeding 41th AIAA/ASME/ASCE/AHS/ASC Structures, Structural Dynamics, and Materials Conference, Atlanta, USA, 2000; AIAA 2000-1573.

13. Craig RR, Kurdila AJ. Fundamentals of Structural Dynamics. Wiley: New York, 2006.

14. Klerk DD, Rixen DJ, Voormeeren SN. General framework for dynamic substructuring: history, review, and classification of techniques. AIAA Journal 2008; 46(5):1169-1181.

15. Kim JG, Lee KH, Lee PS. Estimating relative eigenvalue errors in the Craig-Bampton method. Computers \& Structures 2014; 139:54-64.

16. O'Callahan J. A procedure for an improved reduced system (IRS) model. Proceeding the 7th International Modal Analysis Conference, CT, Bethel, 1989; 17-21.

17. Friswll MI, Garvey SD, Penny JET. Model reduction using dynamic and iterated IRS techniques. Journal of Sound and Vibration 1995; 186:311-323.

18. Xia Y, Lin R. A new iterative order reduction (IOR) method for eigensolutions of large structures. International Journal for Numerical Methods in Engineering 2004; 59(1):153-172.

19. Park KC, Kim JG, Lee PS. A mode selection criterion based on flexibility approach in component mode synthesis. Proceeding 53th AIAA/ASME/ASCE/AHS/ASC Structures, Structural Dynamics, and Materials Conference, Hawaii, USA, 2012; AIAA 2012-1883.

20. Dvorkin EN, Bathe KJ. A continuum mechanics based four-node shell element for general nonlinear analysis. Engineering Computations 1984; 1(1):77-88.

21. Bathe KJ, Dvorkin EN. A formulation of general shell elements-the use of mixed interpolation of tensorial components. International Journal for Numerical Methods in Engineering 1986; 22(3):697-722.

22. Lee Y, Lee PS, Bathe KJ. The MITC3+ shell finite element and its performance. Computers \& Structures 2014; 138:12-23.

23. Jeon HM, Lee PS, Bathe KJ. The MITC3 shell finite element enriched by interpolation covers. Computers \& Structures 2014; 134:128-142.

24. Hurty W. A criterion for selecting realistic natural modes of a structure. Technical Memorandum, Jet Propulsion Laboratory, Pasadena, CA, 1967; 33-364.

25. Kim JG, Lee PS. An accurate error estimator for Guyan reduction. Computer Methods in Applied Mechanics and Engineering 2014; 279:1-19. 